\title{
Premature ejaculation and its management
}

\author{
L S Malavige ${ }^{1,2}$, S Jayawickrema ${ }^{3}$ \\ Sri Lanka Journal of Diabetes, Endocrinology and Metabolism 2015; 5: $13-19$
}

\section{Introduction}

Premature Ejaculation (PE) is a condition in which a man ejaculates earlier than he or his partner would like him to, causing distress to either or both partners. It is also known as rapid ejaculation, or premature climax.

$\mathrm{PE}$ is the most common type of male sexual dysfunction, affecting $14-30 \%$ of males aged 18 years and over $(1,2)$. According to recently published Oxford Sexual Dysfunction Study, men of South Asian origin living in the UK have shown to have a higher prevalence of PE compared to Caucasians (3). PE has shown to be associated with negative personal consequences such as distress, bother, frustration and the avoidance of sexual intimacy. Both affected men and their partners report interpersonal difficulties and an overall reduction in their quality of life. Despite significant distress, many suffer in silence; only a minority seeks professional help, owing to the sensitiveness of the issue, and lack of attention paid by the clinicians towards sexual issues.

\section{Definition and classification of PE}

There is no universally accepted definition for PE. According to the most accepted definition given by International Society for Sexual Medicine (ISSM) three criteria have to be met to diagnose PE. Those are (i) a short ejaculatory latency - the time from penetration to ejaculation; Intra vaginal Ejaculatory Latency Time (IELT) should be less than one minute, however, IELT between one to two minutes can be considered short if it causes significant distress either to the man or his partner. (ii) a lack of perceived self-efficacy or control about the timing of ejaculation; and (iii) distress and interpersonal difficulty related to the ejaculatory dysfunction.

Over the years, diverse PE classifications have been introduced. It could be broadly classified as lifelong PE (primary) or acquired (secondary) PE (4). Men with lifelong $\mathrm{PE}$ experience rapid ejaculation in all sexual encounters including masturbation, and with all partners, which begins when a man first becomes sexually active. Men with acquired PE develop the problem later in life after having a normal ejaculatory latency time previously. Anteportal ejaculation (ejaculation happening before vaginal penetration) is considered the most severe form of PE.
Between $5 \%$ to $20 \%$ of lifelong PE men suffer from anteportal PE (5), such men/couples typically present when they are having difficulty conceiving.

In addition, 'Natural Variable PE' is a condition in which ejaculation happens too rapidly only in certain sexual situations or acts. This may represent a variation of natural ejaculatory function with some subjective sense of diminished control of ejaculation. 'Premature like Ejaculatory Dysfunction' is a condition where a man is distressed about the ejaculatory latency time, despite normal IELT exceeding two minutes (6). In multinational studies, average time from penetration to ejaculation is 5 to 6 minutes with a median IELT of 5.4 minutes (range, 0.55-44.1 minutes) (7).

In clinical practice, self estimation of the ejaculatory time by the patient and the partner is recommended for the assessment of IELT, which is also used to monitor the success of PE treatment. Use of stopwatch to determine the IELT is used in clinical trials. In addition, several questionnaires are available for aiding the diagnosis of $\mathrm{PE}$ and monitoring treatment change or outcome measures. Despite the presence of the diagnostic tools a diagnosis of PE should only be made following a full sexual history taken by a clinician.

\section{Etiology of PE}

It was previously thought that this condition is primarily due to psychological factors. Over the last two decades many biological factors for PE have been described. In certain cases, PE is more complicated and involves a complex interaction of both psychological and biological factors. Genetic predisposition plays a role in most men with lifelong PE (8).

\section{Psychological causes}

There are diverse psychological factors that either predispose or augment PE. Certain personality traits are more vulnerable to PE. Anxiety regarding sexual function is a common precipitating factor. In some, early sexual experiences which may have established a particular pattern that becomes difficult to change later in life could predispose to PE. Examples of such situations are hurry-

${ }^{1}$ Asiri surgical hospital, Sri Lanka, ${ }^{2}$ Oxford center for diabetes, endocrinology and metabolism, university of Oxford, UK, ${ }^{3}$ Medical clinic, out patient department, teaching hospital Kurunegala, Sri Lanka. 
ing to reach a climax in order to avoid being discovered by others or due to a feeling of guilt. Other factors that could have a negative impact on one's sexual performance are history of sexual abuse, attitudes towards sex among peers and some personal factors such as poor body image, depression or problems in the relationship (9). Some of the psychosexual issues leading to PE can be less obvious. Men affected by PE have shown significantly low selfesteem and self confidence than their non-PE counterparts.

Emotional or mental strain in any area of one's life can play a role in PE, often limiting one's ability to relax and focus during sexual encounters. If one has previously had satisfying sexual relationships in which PE happened infrequently or not at all, it is likely that current interpersonal issues between him and his partner are contributing to the problem. In such cases, relationship issues and female sexual dysfunctions such as anorgasmia, hypoactive sexual desire disorder, sexual aversion, sexual arousal disorders, and sexual pain disorders such as vaginismus could be causative factors.

\section{Biological causes}

A number of biological factors may contribute to PE, including, abnormal testosterone or prolactin levels (10), disturbances in central serotonergic neurotransmission (11), abnormal reflex activity of the ejaculatory system, penile hypersensitivity (12), hyper-arousability, a higher cortical representation of the pudendal nerve, erectile difficulties (13), prostatitis/ chronic pelvic pain syndrome (14), detoxification from prescribed drugs e.g. raboxetine, trifluoperazine or recreational drugs (narcotics) (15), chronic pelvic pain syndrome (16), thyroid disorders (17) and genetic factors.

A high prevalence of lifelong PE among first degree relatives has been shown in a study among Dutch men with lifelong PE (18). Genetic study to demonstrate the genetic predisposition was performed by Janssen et al which demonstrated an association of the 5-HTLPR gene polymorphism and the IELT (19).

$\mathrm{PE}$ is common in mild to moderate erectile dysfunction (3). This could be due to men who are anxious about obtaining or maintaining their erection during sexual intercourse forming a pattern of rushing to ejaculate before losing erection. Therefore men who have PE secondary to erectile problems, need careful evaluation in order to identify the cause for their erectile dysfunction. In such men, treating erectile problem will often improve ejaculatory time.

\section{Clinical assessment}

As PE is a clinical diagnosis, a full clinical history and a focused physical examination play an important role in its diagnosis. Nevertheless, as the patients may be embarrassed and shy when relating details of their sexual experiences, clinical assessment can be challenging. As it is more an issue of a couple, it is useful to involve the partner in the consultation. In diagnosis, the three main components of PE should be addressed; ie. timing, control and distress associated with the condition.

The stopwatch assessment of ejaculatory time is not recommended in routine clinical management due to potential disruption of sexual pleasure. It is usually used in clinical trials and observational studies. ISSM recommends to use self-estimation by the man or his partner as the accepted method of determining IELT in clinical practice (20).

There are 3 recommended questionnaires used to diagnose and evaluate PE. Premature Ejaculation Diagnostic Tool (PEDT) is developed specifically to be used as a screening questionnaire based on DSMIV-TR criteria, whereas the Premature Ejaculation Profile (PEP) and Index of Premature Ejaculation (IPE) are used to measure the treatment outcome (21).

Establishing PE diagnosis, diagnostic subtype and its impact on the relationship with the partner and the quality of life is important in perceiving the scope of the problem and to determine management plan. In clinical practice this is established with detailed sexual history. Symonds $\mathrm{T}$ et al has developed questions as a guide to establish the diagnosis and impact of PE (22) (Table 1).

For lifelong PE, a physical examination is advisable but not compulsory. In cases of acquired PE, a targeted physical examination is mandatory to find out associated problems, such as risk factors for ED, thyroid dysfunction, and prostatitis. Apart from the general examination a focused examination of the genitalia, digital rectal examination for the prostate is recommended to exclude prostatitis. Laboratory and other physiological tests are rarely indicated. History and examination are often sufficient to reach a diagnosis.

\section{Treatment}

All men need careful evaluation before treatment in order to achieve better outcome. Treatment options for premature ejaculation include psychosexual counselling, behavior therapy and medications; guided by the type of PE (lifelong/acquired), bio-psycho-social assessment and patient preference. For many men, a combination of these treatments work best. Nonetheless, according to ISSM Standard Operating Procedures all men seeking treatment for PE should receive basic psychosexual counselling. Inclusion of the partner in the treatment process is important for treatment success though it is not mandatory. The management algorithm of PE is given in figure 1. 
Table 1. Useful questions in clinical assessment

Recommended questions

for diagnosis

Optional questions -

Differentiate lifelong and acquired $\mathrm{PE}$

Optional questions -

Assess erectile function

Optional questions -

Assess relationship impact

Previous treatment

Impact on quality of life
- What is the time between penetration and ejaculation (coming)?

- Can you delay ejaculation?

- Do you feel bothered, annoyed and/or frustrated by your premature ejaculation?

- When did you first experience premature ejaculation?

- Have you experienced premature ejaculation since your first sexual experience on every/almost every attempt and with every partner?

- Is your erection hard enough to penetrate?

- Do you have difficulty in maintaining your erection until you ejaculate during intercourse?

- Do you ever rush intercourse to prevent loss of your erection?

- How upset is your partner with your premature ejaculation?

- Does your partner avoid sexual intercourse?

- Is your premature ejaculation affecting your overall relationship?

- Have you received any treatment for you premature ejaculation previously?

- Do you avoid sexual intercourse because of embarrassment?

- Do you feel anxious, depressed or embarrassed because of your premature ejaculation?

\section{Psychotherapy and behavioral therapy}

Psychotherapy includes both psychosexual counselling and relationship or marital counseling. This usually involves both the male patient and the partner. Psychosexual counselling includes improving awareness on the prevalence of PE and the average IELT of the general population, and expanding the diversity of sexual activities of the couple thus preventing the avoidance of sexual activities. Psychosexual counselling also include increasing man's confidence in sexual performance, and his overall self-confidence, lowering performance anxiety; increasing communication with his partner, and unravelling issues between the couple which may have precipitated PE. Men with Premature-Like Ejaculatory Dysfunction require psychotherapy whereas men with Natural Variable $\mathrm{PE}$ should be provided necessary information and reassured.

\section{Behavioural therapy}

The 'stop-start' technique and the 'squeeze' technique has been used since 1950s but with no conclusive long-term results (23). These two techniques are designed to make the man aware when he approaches ejaculatory inevitability which involves stopping of the stimulation and resumption once the arousal subsides. In addition learning to relax the body and certain sexual positions such as women on top position or lateral position can be helpful in controlling ejaculation. Pre-coitus masturbation is used by younger men suffering from PE, as a method of partial desensitization of the penis but this is not an acceptable method for many (24).

However, if partners are not involved in treatment, they may be resistant to changing the sexual interaction. A cooperative partner can enhance the man's selfconfidence, sexual techniques, self-esteem, and sense of masculinity and assist the man to develop ejaculatory control. These measures will improve PE, as well as sexual relationship and the broader aspects of their relationship.

\section{Pharmacological therapy}

In the current context, pharmacological management of PE is through drug groups including antidepressants, local anaesthetic agents and phosphodiesterase type 5 inhibitors. Yet at present only Dapoxetine is licensed for the treatment of PE. While some drugs are administered 
Figure 1. Premature ejaculation - ISSM management algorithm

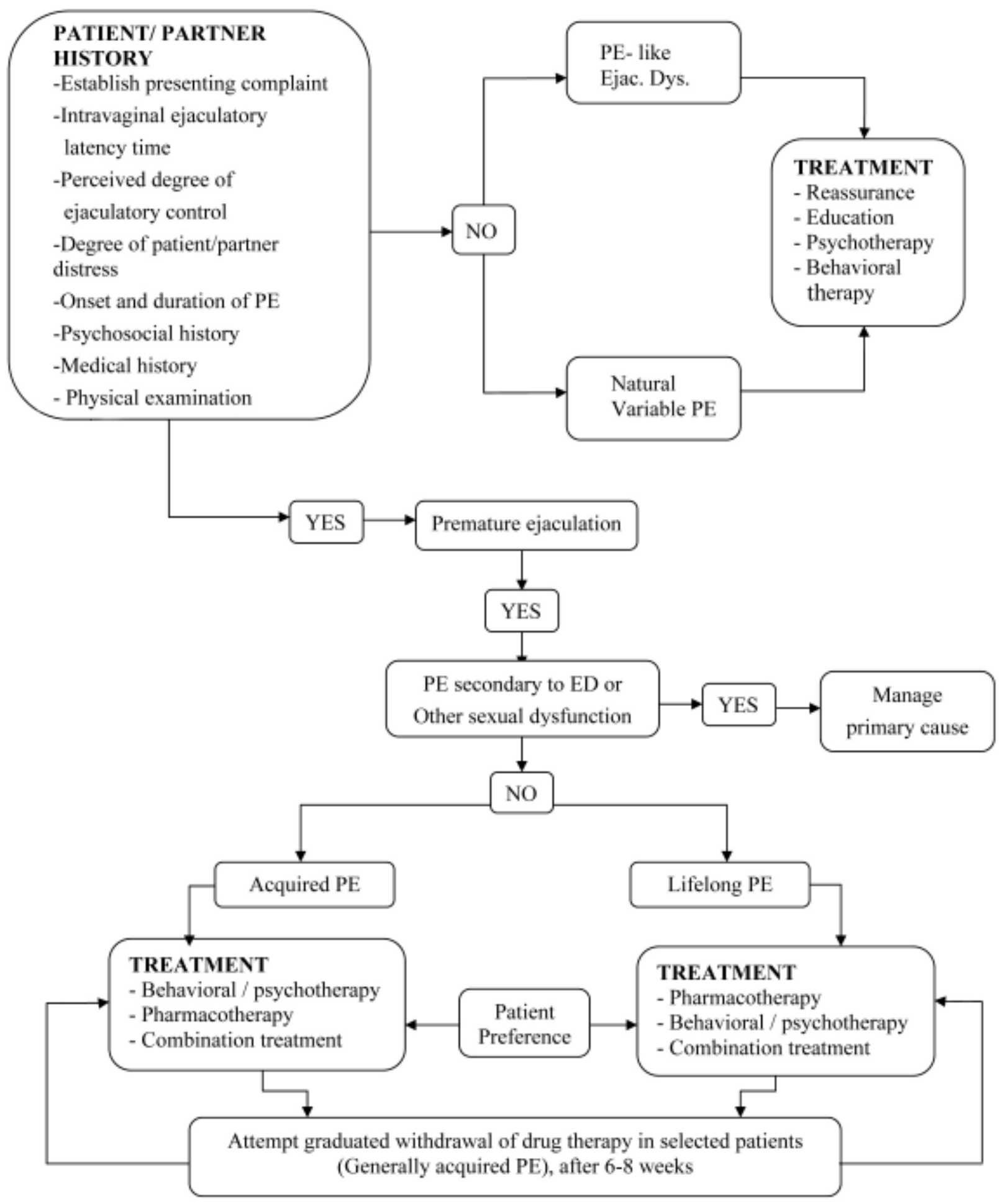

on demand others are administered daily. An on demand drug offers the advantage of less side effects of the drug whereas the daily dosage allows spontaneous sex but more pronounced side effects of the drug.

\section{Desensitizing agents}

The most conventional type of pharmacological therapy is desensitizing or local anaesthetic agents. 
Desensitizing or local anaesthetic agents used to delay ejaculation include lidocaine, prilocaine and benzocaine which come in different forms such as creams and sprays (25). However as the application of a cream is cumbersome due to the requirement of a condom to minimize its anaesthetizing effects on the vagina, aerosol sprays have become more popular. The sprays are odourless, adherent solutions that act within 5 minutes by penetrating the glans, without penetrating intact keratinized skin, making them more user friendly. Hypoanaesthesia of the penile shaft is a common side effect which in some men can affect erection. Local irritation is rare.

\section{Oral drug therapy}

Monoamine oxidase (MAO) inhibitors and alphablockers were the first oral medication used for the treatment of PE, which are not used for PE treatment anymore. However, selective alpha-blockers like Terazosin have shown to be effective in treating PE in patients with concurrent lower urinary tract symptoms (26). More recently, drugs acting on central serotonergic neurons by blocking the axonal reuptake of serotonin from synaptic clefts and thereby enhancing the neurotransmission (including Selective Serotonin Reuptake Inhibitors and Tricyclic Antidepressants) have transformed pharmacological therapy.

\section{Antidepressants}

Clomipramine is a Tricyclic Antidepressant (TCA) that increases the IELT in patients with PE both through continuous and on-demand dosing (27). Main side effects of clomipramine include fatigue, dizziness, dry mouth and hypotension.

Selective Serotonin Reuptake Inhibitors (SSRIs) such as escitalopram, fluoxetine, fluoxamine, sertraline and paroxitine are serotonergic agents that work by activation of the $5-\mathrm{HT}^{2 \mathrm{C}}$ receptor (28). With the use of SSRIs the IELT is increased between twofold and eightfold (29). Paroxetine produced the best ejaculatory delay, increasing IELT by 8.8 fold from the baseline (29).

In daily dosage, paroxetine $10-40 \mathrm{mg}$, clomipramine $12.5-50 \mathrm{mg}$, sertraline $50-200 \mathrm{mg}$, fluoxetine $20-40 \mathrm{mg}$ or citalopram 20-40 mg is effective in expanding the IELT(3033). Although the full therapeutic effect takes 2-3 weeks to ensue, usually the ejaculation delay occurs within few days of commencing treatment. On demand administration of clomipramine, paroxetine, sertraline, and fluoxetine 4-6 hours before intercourse is moderately efficacious and better tolerated, but results in considerably less ejaculatory delay than daily dosing (34). On-demand dosing can be combined with either an initial trial of daily treatment or concomitant low dose daily treatment.

Adverse effects with SSRIs are nausea, fatigue, yawning, perspiration and loose stools which usually resolve in 2-3 weeks. SSRIs should be avoided in patients with a history of bipolar depression (35). It is essential to perform follow-up assessments to evaluate the efficacy of the drug as well as for the potential side effects, especially regarding associated sexual dysfunction and suicide risks. Furthermore, due to the risk of SSRI withdrawal syndrome, patients should be advised to avoid abrupt cessation or dose reduction without medical advice (36). Treatment with SSRIs had shown a lower incidence of side effects than clomipramine (37).

Out of all the available medication, Dapoxetine, a rapid acting and short half-life, potent SSRI is the only licensed drug for the treatment of PE and is used as an on-demand drug. Dapoxetine is structurally similar to fluoxetine and has a $\mathrm{T}_{\max }$ of 1.4-2.0 hours and a terminal half life of 19 hours. It is taken only when needed, 1-3 hours before sexual intercourse is anticipated (38). It is extensively metabolized in the liver and eliminated in urine. Therefore it is contraindicated to be administered with potent CYP3A4 inhibitors such as ketoconazole and have to be cautious in co administration with moderate CYP3A4 inhibitors and potent CYP2D6 inhibitors such as fluoxetine. Dapoxetine has a relatively mild side effect profile including nausea, diarrhea, headache and dizziness whilst proven to be effective for the treatment of PE. It has shown to increase the IELT by 3 folds and effective on both men with lifelong and acquired PE (39). At the time of writing Dapoxetine is not registered in Sri Lanka.

\section{PDE5 inhibitors}

Sildenafil, vardenafil, tadalafil are PDE5 inhibitors used in the management of PE when it is associated with erectile dysfunction. Since a third of men with ED also suffer from PE, the association between PE and ED may be explained by the fact that when a man suffers from ED, he makes an effort to achieve ejaculation before he loses the erection, leading to PE (40). PDE5 inhibitors are ineffective in men with normal erectile function.

\section{Tramadol}

Tramadol, a centrally acting opiate is used to treat PE though the exact mechanism of action is not known. It's efficacy has been proved in studies with a 2.9 fold increase in IELT specifically as an on-demand medication (41). However due to the limited data availability on its safety, and the potential for addiction, the use of tramadol in PE treatment is limited to specialists.

The newest addition to the management of $\mathrm{PE}$ is the delay device of which the efficacy was shown in a small clinical trial involving 52 patients. There is no evidence to support use, intra-cavernorsal injections, selective dorsal nerve neurotomy or hyaluronic acid gel glans penis augmentation in management of PE. 


\section{Conclusion}

Knowledge on physiology and management of PE has changed drastically over last two decades. Despite the lack of controlled clinical trials, a wide range of psychological and behavioural interventions have been developed for the treatment of PE. In men with acquired $\mathrm{PE}$, identifying and correcting underlying causes such as erectile dysfunction, hyperthyroidism, prostatitis, psychological and relationship issues are important. Premature-Like Ejaculatory Dysfunction and Natural variable PE often needs only behavioural therapy and psychosexual counselling. For life long and acquired PE pharmacotherapy is often indicated. In addition to increasing IELT, psychosexual and behavioural therapies help improve the relationship, self confidence and sexual confidence.

The pharmacotherapy can be classified as daily dosing and on demand dosing. The use of SSRIs have revolutionised the management of PE. There is level 1a evidence to support the efficacy and safety of on-demand dosing of dapoxetine for the treatment of lifelong and acquired PE. Off-label daily dosing of the SSRIs paroxetine, sertraline, citalopram, fluoxetine, and the serotonergic tricyclic, clomipramine has also shown level 1a evidence. There is level 1a evidence for off-label on-demand dosing of clomipramine, paroxetine, and sertraline in the management of PE. The use of topical local anaesthetics such as lidocaine and/or prilocaine as a cream, gel, or spray is well established and is moderately effective in delaying ejaculation (level 1b). PDE5 inhibitors are indicated in the treatment of premature ejaculation only when there is co-existing erectile dysfunction.

\section{References}

1. Patrick DL, Althof SE, Pryor JL, et al. Premature ejaculation: an observational study of men and their partners. $J$ Sex Med 2005; 2: 358-67.

2. Aschka C, Himmel W, Ittner E, and Kochen MM. Sexual problems of male patients in family practice. J Fam Pract 2001; 50: 773.

3. Malavige LS, Wijesekara P, Seneviratne ED, Ranasinghe P, Levy JC. Ethnic Differences in Sexual Dysfunction among Diabetic and Nondiabetic Males: The Oxford Sexual Dysfunction Study. Journal of Sexual Medicine 2013; 10: 500-8.

4. Godpodinoff ML. Premature ejaculation: clinical subgroups and etiology. J Sex Marital Ther 1989; 15:130.

5. Pagani E, Rodrigues O, Torselli M, Genari D. Characterization of 305 Men With Complaints of Premature Ejaculation. International Journal of Impotence Research 1996; 8: 172.

6. Waldinger MD, Schweitzer DH. Changing paradigms from a historical DSM-III and DSM-IV view toward an evidence-based definition of premature ejaculation. Part
II-Proposals for DSM-V and ICD-11. J Sexual Med 2006; 3: 693-705.

7. Waldinger MD, Quinn P, Dilleen M, Mundayat R, Schweitzer DH, Boolell M. A multinational population survey of intravaginal ejaculation latency time. The Journal of Sexual Medicine 2005; 2(4): 492-7.

8. Sadeghi-Nejad H, Watson R. Premature ejaculation: current medical treatment and new directions (CME) J Sex Med 2008; 5(5):1037-50; quiz 1051-2.

9. Althof S, Leiblum S, Chevret M, et al. Psychological and Interpersonal Dimensions of Sexual Function and Dysfunction. J Sex Med 2005; 2(6): 793-800.

10. Corona G, Jannini EA, Mannucci E, et al. Different testosterone levels are associated with ejaculatory dysfunction. J Sex Med 2008; 5: 1991-8.

11. Waldinger M, Berendsen HH, Blok BF, Olivier B, Holstege G. Premature ejaculation and serotonergic antidepressantsinduced delayed ejaculation: the involvement of the serotonergic system. Behavioral Brain Research 1998; 92: 111-8.

12. Xin Z, Choi Y, Rha K. Somatosensory evoked potentials in patients with primary premature ejaculation. Journal of Urology 1997; 158: 451-55.

13. Chia S. Management of premature ejaculation: A comparison of treatment outcome in patients with and without erectile dysfunction. International Journal of Andrology 2002; 25: 301-5

14. Screponi E, Carosa E, Di Stasi SM, Pepe M, Carruba G, Jannini EA. Prevalence of chronic prostatitis in men with premature ejaculation. Urology 2001; 58: 198-202.

15. Peugh J, Belenko S. Alcohol, drugs and sexual function: a review. Journal of Psychoactive Drugs 2001; 33: 223-32.

16. Gonen M, Kalkan M, Cenker A, Ozkardes H. Prevalence of premature ejaculation in Turkish men with chronic pelvic pain syndrome. Journal of Andrology 2005; 26: 601-3.

17. Carani C, Isidori AM, Granata A, et al. Multicenter Study on the Prevalence of Sexual Symptoms in Male Hypo- and Hyperthyroid Patients. Journal of Clinical Endocrinology and Metabolism 2005; 90: 6472-79.

18. Waldinger M, Rietschel M, Nothen N, Hengeveld MW, Olivier B. Familial occurrence of primary premature ejaculation. Psychiatric Genetics 1998; 8: 37-40.

19. Janssen P, Bakker S, Rethelyi J, et al. Serotonin transporter promoter region (5-HTTLPR) polymorphism is associated with the intravaginal ejaculation latency time in Dutch men with lifelong premature ejaculation. Journal of Sexual Medicine 2009; 6: 276-84.

20. Pryor JL, Broderick GA, Ho KF, Jamieson C, Gagnon D. Comparison of estimated versus measured intravaginal ejaculatory latency time in men with and without premature ejaculation. Journal of Sexual Medicine 2005; 3: 54.

21. Symonds T, Perelman M, Althof S, et al. Further evidence of the reliability and validity of the premature ejaculation diagnostic tool. International Journal of Impotence Research 2007; 19: 521-25. 
22. Symonds T, Roblin D, Hart K, Althof S. How does premature ejaculation effect a man's life. Journal of Sex and Marital Therapy 2003; 29: 361-70.

23. Hawton K, Catalan J, Martin P, Fagg J. Long-term outcome of sex therapy. Behav Res Ther 1986; 24: 665-75.

24. Sadeghi-Nejad H, Watson R. Continuing medical education: premature ejaculation: current medical treatment and new directions (CME). J Sexual Med 2008; 5: 1037-50.

25. Carson C, Wyllie M. Improved ejaculatory latency, control and sexual satisfaction when PSD502 is applied topically in men with premature ejaculation: results of a phase III, double-blind, placebo-controlled study. J Sex Med 2010; 7: 3179-89.

26. Basar M, Yimaz E, Ferhat M, Basar H, Batislam E. Terazosin in the treatment of premature ejaculation: a shortterm follow-up. Int Urol Nephrol 2005; 37: 773-7.

27. Strassberg D, Brazao CdG, Rowland D, Tan P, Slob A. Clomipramine in the treatment of rapid (premature) ejaculation. J Sex Marital Ther 1999; 25: 89-101.

28. Waldinger M, Hengeveld M, Zwinderman A, Olivier B. Effect of SSRI antidepressants on ejaculation: a doubleblind, randomized, placebo-controlled study with fluoxetine, fluvoxamine, paroxetine, and sertraline. J Clin Psychopharmacol 1998; 18: 274-81.

29. Waldinger MD, Zwinderman AH, Schweitzer DH, Olivier B. Relevance of methodological design for the interpretation of efficacy of drug treatment of premature ejaculation: a systematic review and metaanalysis. International Journal of Impotence Research 2004; 16: 369-81.

30. McMahon CG. Treatment of premature ejaculation with sertraline hydrochloride: A single-blind placebo controlled crossover study. J Urol 1998; 159:1935-8.

31. Atmaca M, Kuloglu M, Tezcan E, Semercioz A. The efficacy of citalopram in the treatment of premature ejaculation: A placebo-controlled study. Int J Impot Res 2002; 14: 502-5.

32. Kara H, Aydin S, Yucel M, Agargun MY, Odabas O, Yilmaz Y. The efficacy of fluoxetine in the treatment of premature ejaculation: A double-blind placebo controlled study. J Urol 1996; 156: 1631-2.
33. Waldinger MD, Hengeveld MW, Zwinderman AH. Paroxetine treatment of premature ejaculation: A doubleblind, randomized, placebo-controlled study. Am J Psychiatry 1994; 151: 1377-9.

34. Waldinger MD, Zwinderman AH, Olivier B. On-demand treatment of premature ejaculation with clomipramine and paroxetine: A randomized, double-blind fixed-dose study with stopwatch assessment. Eur Urol 2004; 46: 510-5.

35. Marangell LB, Dennehy EB, Wisniewski SR, et al. Casecontrol analyses of the impact of pharmaco-therapy on prospectively observed suicide attempts and completed suicides in bipolar disorder: Findings from STEP-BD. $J$ Clin Psychiatry 2008; 69: 916-22.

36. Black K, Shea CA, Dursun S, Kutcher S. Selective serotonin reuptake inhibitor discontinuation syndrome: Proposed diagnostic criteria. J Psychiatry Neurosci 2000; 25: 255-61.

37. Kim SC, Seo KK. Efficacy and safety of fluoxetine, sertraline and clomipramine in patients with premature ejaculation: a double-blind, placebo controlled study. J Urol 1998; 159: 425-27.

38. McMahon C, Kim S, Park N, et al. Treatment of Premature Ejaculation in the Asia-Pacific Region: Results From a Phase III Double-blind, Parallel-group Study of Dapoxetine. Journal of Sexual Medicine 2009; 7: 256-68.

39. Porst H, McMahon C, Althof S, Sharlip I, Bull S, Rivas DA. Baseline characteristics and treatment outcomes for men with acquired or lifelong premature ejaculation with mild or no erectile dysfunction: Integrated analysis of two phase III dapoxetine trials. Journal of Sexual Medicine 2010; 7(6): 2231-42.

40. Corona G, Petrone L, Mannucci E, et al. Psycho-biological correlates of rapid ejaculation in patients attending an andrologic unit for sexual dysfunctions. Eur Urol 2004; 46: 615-22.

41. Alghobary MMD, El-Bayoumy YMD, Mostafa YMD, Mahmoud EHMMD, Amr MMD. Evaluation of tramadol on demand vs. daily paroxetine as a long-term treatment of lifelong premature ejaculation. J Sexual Med 2010; 7: 2860-7. 